\section{Interstitial Deletion 46,XY,del(1)(q23q25)}

Section of Human Genetics, Department of Obstetrics and Gynecology, Northwestern University Medical School, Chicago, IL 60611, USA

A de novo interstitial deletion [46,XY, del(1)(pter $\rightarrow \mathrm{q} 23:: \mathrm{q} 25$ $\rightarrow$ qter)] was detected in a $1620-\mathrm{g}$ infant born after 40 weeks gestation to an 18-year-old mother of Polish descent and a 23-year-old father of Hispanic descent. The father had worked in a chemical factory for $1 \frac{1 / 2}{2}$ years prior to conception of the proband. The proband's only sib, a female, is said to be normal. Gestation was characterized by intrauterine growth retardation and oligohydramnios. The infant showed facial dysmorphisms reminiscent of trisomy 13 (sloping forehead, broad nasal bridge, bilateral cleft lip and palate), but no eye anomalies were evident. Other anomalies included brachydactyly, bilateral clinodactyly $\mathrm{V}$, left distal transverse palmar crease, and bilateral equinovarus. No other anomalies were revealed by roentgenographic or ultrasonographic studies, including those of the kidneys and head. Nonetheless, the infant developed renal failure and died 6 days after birth in cardiac arrest. Necropsy was not permitted.

The interstitial deletion was present in all 58 (GTG-banded) metaphases derived from cord blood cultures (lymphocytes) and in all 18 metaphases derived from fibroblast cultures. Chromosomal complements of both parents were normal. Clinical features, prognosis, and associated risk factors characteristic of autosomal interstitial deletions need to be elucidated. We would therefore be interested in corresponding with others who have observed interstitial deletions involving chromosome no. 1.

\section{A New Case of Satellited Yq Chromosome}

\author{
M. J. Martín, T. Rodriguez, and J. A. Abrisqueta \\ Instituto de Genética (C.S.I.C.), Velázquez, 144, Madrid-6, Spain
}

We have found a satellited Yq chromosome in a 20-year-old boy with clinical hypogonadism and minor abnormal traits such as discrete brachydactyly, cubitus valgus, and bone age of 13.5 years. His normal father carried the same marker chromosome. The G-, C-, and Q-bands confirmed the small size of the Yqs chromosome which does not show its typical fluorescent band. NOR-bands demonstrated the presence of heavily stained nucleolar organizing regions on the $\mathrm{Yq}$. We suggest that the translocation from which the Yqs originated may have occurred in the first meiotic division. A variable phenotypic expression has been observed in the Yqs carriers. It appears that the satellited $Y$ chromosome may be associated with minor clinical deviations and in some cases it may favour meiotic nondisjunction.

We would like to receive from or to send to colleagues full information for eventual joint publication on similar cases. 